\title{
Standards for Integrated Services and Networks
}

\author{
J P Chester \& K R Dickerson \\ RACE Consensus Management Office \\ 165 boulevard du SOUVERAIN \\ B-1160 Brussels
}

Tel: +3226748522

Fax: +3226748538

Email:joe@ric.iihe.ac.be

\begin{abstract}
This paper discusses the technical requirements and the standards that are required before global services can be implemented across multiple network operator and service provider domains in Europe. Two advanced service scenarios are described to illustrate the sort of global services that are required, and the problems of implementing these using current technology are discussed. The most important standards bodies for solutions to these problems are then identified.
\end{abstract}

\section{Keywords}

IN, Multimedia, Network Architecture, Personal Mobility, Private and Public Networks, Services, Signalling, Standards, Terminal Mobility, TMN, VPN.

\section{Introduction}

The telecommunications market in Europe is seeing a proliferation of service providers and network operators. In order to provide common services to customers across the range of network operator and service provider domains it is necessary to provide standards that will allow interconnection and interoperability between networks and services across Europe.

This paper describes the problems that currently prevent the provision of global services, and 
introduces the standards that are required to allow interconnection of networks and interoperability between services.

\section{Scenarios for Advanced Service Provision}

Scenarios are a useful way to present advanced applications and services in order to estimate the likely demand. Otherwise it may be difficult to visualise the capabilities of applications and services which it is impossible to personally experience. Scenarios are also a useful way to stimulate discussion on a wide range of issues associated with the application or service, ranging from usage and usability issues through to the implications for service creation, management and services platforms.

The scenarios described here cover PSCS (Personal Services Communications Space) and Hypermedia. They represent ends of a spectrum of service opportunities that provide on the one hand capabilities that will allow users to communicate with each other independent of their physical location, and on the other the ability to easily access a wide range of information sources at a wide range of bit rates. The combination of these two scenarios with a user-friendly interface would provide the holy grail where instant access can be obtained to people or information anywhere in the world.

\subsection{PSCS}

This scenario was developed by the MOBILISE project [3] and is based on a development of the UPT concept as defined by ETSI [4]. It is based around the concept of personal mobility. - the user can move between geographical locations and can still be contacted on a pre-specified number.

Key concepts in this scenario are personal numbering, number portability, and personalisation and customisation of services. Personal communication offers the ability to communicate in different roles and to organise communication according to the user's preferences. Users can play different roles and set up different routings for calls depending on the caller, the time of day and other requirements. The link with mobile services is extremely important because customers will want to access these services via mobile as well as fixed terminals.

\subsection{Hypermedia}

The second scenario is based on the concept of a global village, sometimes referred to as cyberspace, a space full of information objects. Multimedia is already bringing the ability to see, hear (and eventually smell) your colleagues remotely, as well as to view and point at shared objects on screen. This concept is extended through the use of explicit links between multimedia objects to become hypermedia. This provides the ability to sit at a terminal and set up instant video connections to colleagues and experts and to access all the world's knowledge in a variety of media.

The key to this scenario is high quality video, voice and data communications with fast response times. It requires high bit rates and generally makes greater use of multimedia and multipoint services than the PSCS scenario. 


\section{Barriers to Implementation}

Today there are a number of barriers to providing these sorts of services, especially where they must be provided globally across a range of network operator and service provider domains. These problems were investigated by the ETSI DASH Task Group which reported in May 1994 [1]. Problems identified include:

- The difficulty of interworking between public and private networks and services. The provision of services such as VPN depend on interworking capabilities between public and private domains. Regulatory developments are also likely to lead to the breaking down of the traditional barriers between public and private domains, and will heighten the need for convergence between the two sectors.

- The difficulty of interworking between fixed and mobile services. Different architectures are currently used for fixed and mobile areas. This may prevent similar services being offered across the two environments.

- The difficulty of creating and managing distributed services in an IN structured environment. There may also be a problem of interoperability between similar services created using different service creation environments.

The problems currently prevent supplementary services available in a private domain (such as a PBX) from being extended transparently over a public network or to a mobile terminal. This will be even more the case in the future with a greater range of $\mathrm{IN}$-supported services.

\section{Standards Required for Implementation}

The layered model in Figure 1 can be used to illustrate the type of standards issues. There are important issues at all layers in the model, from the application layer down through the service infrastructure, transport and network access layers, down to the physical transmission layer. All of these must be correct and interwork satisfactorily in different environments in order to offer effective services to customers. 


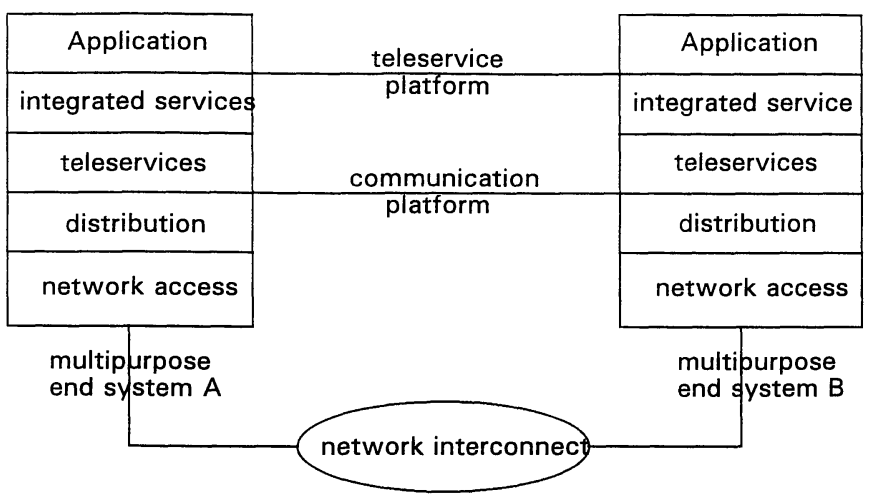

Figure 1 Layered model of telecommunications service provision.

Functions required for service implementation can be provided in either the terminal or the network and must be complementary.

The priority work areas that will need standards to be developed to overcome these barriers are described in the remainder of this section.

\subsection{Service and network capability description}

Service and network architecture description methods are required to ensure the consistent description of services specified for different platforms, and to allow services to be described in a network independent fashion. This requires:

- The revision of the I.130 3-stage method [7] to provide network-independent service descriptions at Stage 1, and sufficiency flexibility to cover services requiring broadband, mobile and multimedia capabilities.

- A movement away from rigid service descriptions, as provided by CCITT for ISDN services, and towards the reduced level of specification associated with the IN approach. This will allow a larger range of more flexible services to be provided to customers, based on agreed sets of common network capabilities.

- The use of a common state model as a basis for all service descriptions. This will provide a greater degree of interoperability between services.

Network capabilities to support IN services are being defined in three phases, known as capability sets 1-3. The current schedule for these is as follows: CS1 (1994), CS2 (1996) and CS3 (>1996). The following issues must be addressed:

- It is still to be decided which IN service features will be included in CS2 and CS3. It is important that the necessary capabilities are provided to allow the scenarios described in Section 2 to be implemented.

- How will these services be created and deployed effectively? 
- The evolution of IN towards the distributed platform approach based on ODP that will be required for CS3.

The last two issues are addressed further in the following section.

\subsection{The relationship between TMN and IN}

It is essential that the services required for the implementation of the scenarios described in Section 2 can be created and managed effectively. Key issues relate to the interactions between the Service Creation Environment, the IN Platform, and the TMN. The interactions defined between these entities must allow for market requirements, including both bilateral and multilateral relationships between the various actors. The DASH report [1] represents a useful starting point for defining the requirements of these interactions. The DASH model for the relationship between the TMN and IN is shown in Figure 2. This is oriented towards IN CS1 type services, and highlights the interfaces to the basic call processing state machine.

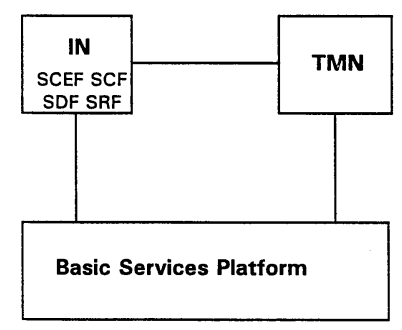

Figure 2 DASH model for the integration of TMN and IN.

The requirements for IN CS2 type services will involve a high degree of distribution of management and control, as well as enhancement of the basic call model to include more advanced services such as network and non-call related services. A refinement of the DASH model, known as the SMP Model, is shown in Figure 3. The main advantage of this model is that it highlights a more important set of interactions for further study, in the context of IN CS2 and CS3 and to meet the objectives of TINA-C. These interactions focus more on the information systems viewpoint, and show clearly the need for detailed study of a number of major issues in the telecommunications services environment as a whole.

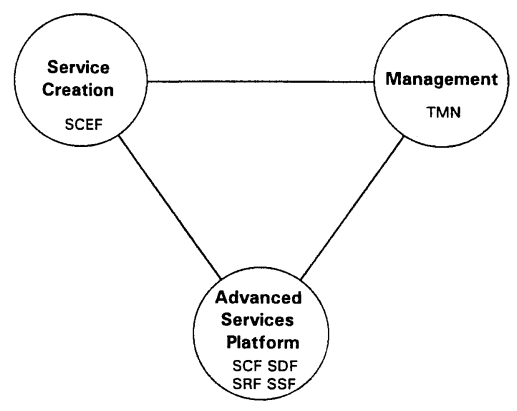

Figure 3 SMP Model for the integration of TMN and IN. 
A more detailed analysis of the use of the SMP model to derive requirements for R\&D and for standardisation activity is given in [5,6]. Some of the key results of the use of the model are presented below.

\subsubsection{Interaction between Service Creation and Management}

Service creation will take place in a non-real time Service Creation Environment, off-line from the services execution platform. As a consequence, there is a need to establish processes and procedures for:

- The effective interaction between the Management entity of the telecommunications services environment and the Service Creation entity. This interaction will govern processes and procedures, as well as deal with the two-way flow of information, and the transfer of service logic, service data, results and performance information.

- The deployment (i.e. the transfer of service logic) via the TMN to the Execution Platform. It is a principle of quality management of the telecommunications services environment that upgrading of the Execution Platform is under Management control. The service data always involves changes to $\mathrm{TMN}$ data.

It is considered important for some types of services that subscribers have a limited ability to customise certain features of the services in accordance with their preferences. Separation of service logic and service data is an important principle. Such service customisation must be under management control, and the functionality of the Management entity will need to make specific provision for this.

\subsubsection{The use of Transaction Processing Technology}

The interactions between the three entities in Figure 3 involve high levels of information transfer and processing. Research is needed on the data consistency aspects of distributed systems control, for current services as well as for distributed IN and TMN type services. The use of Transaction Processing technology seems to be a promising means to achieve this.

\subsubsection{Distributed Control of new classes of services}

The introduction of more advanced services will require distribution of the control and management functions. In addition, the requirement with each change in services for rapid upgrading of the SSP functionality, and of the capabilities of the SCP - SSP and SCP - SCP protocols needs further study. The use of DCE type architectures may provide solutions to some of these issues.

\subsubsection{Building Blocks}

In the Service Creation Environment, to enable rapid and flexible service provision there is a need to construct new services from building blocks. These building blocks should not be standardised, but the interfaces to the building blocks should be clearly specified by manufacturers (e.g. with template descriptions) in order to allow service providers to put together the building blocks in the correct way. This applies to both $\mathrm{IN}$ services and TMN applications. 


\subsubsection{Emerging IT technologies for IN services and TMN applications}

De facto standards, such as X/Open, OMG and OSF, and emerging IT technologies such as DCE, OLTP, CORBA and Motif, are currently a major influence in the management area. Additional effort is needed on the integration of these into the environment for telecommunications services.

\subsubsection{Implications of inter-domain interactions via $X$}

For inter-domain interaction between telecommunications services environments under the control of different administrations (however organised) the only interaction will take place via the $\mathrm{X}$ reference point. The requirements of the telecommunications services environment on this reference point need to be considered, and must include:

- distribution of service logic between domains, or

- distribution of service descriptions to allow interoperable services to be created in different SCEs,

- transfer of service customisation configuration data, and control of the resulting changes.

\subsubsection{The need for Multi-vendor services environments}

In order to ensure that services can be deployed across multi-vendor management and service execution platforms, these platforms need agreed APIs. The output of the service creation activity should be standardised in accordance with these APIs.

\subsection{Personal mobility}

In order to meet the needs of the PSCS scenario, a number of issues need to be resolved and new standards put in place.

PSCS is a distributed service, and as such will reply on an early implementation of IN CS2. One of the key issues is how the distribution aspects are implemented in the Advanced Services Platform.

Another requirement of the PSCS scenario involves the maintenance and updating of customer profile information. This is a management function, and means are needed to implement this requirement through the TMN. In addition, PSCS implies that the management and control of the services are also distributed. An interesting issue is whether there is a need for two different approaches to the distribution issues in both the advanced services platform and the management platform.

Distributed management of resources in different administrative domains has been studied by a number of projects, including RACE PREPARE and EURESCOM P226 and P230. There has been two very useful workshops on UPT management organised by R2083. A Co-operative Model is seen as satisfying the requirements of the current operators for integrity of control over their own resources. Implementation of distributed management of PSCS type services may require implementation of transaction processing schemes between the various domains. 


\subsection{Fixed vs. mobile service interworking}

It is important that services can interwork and can originate and terminate on either fixed or mobile networks. In ETSI UMTS is currently being specified to provide an infrastructure that will support the use of mobile terminals. In ITU this is known as FPLMTS.

\subsection{User interface (service configuration) issues}

The usability of single services has been well investigated during the RACE I and II programmes. The focus is now moving towards the integration and configuration of services to meet user requirements. The concurrent execution of tasks, perhaps using different media and requiring the sharing of screens of multimedia information between participants, must be made simpler for users. Even professional users have problems interacting with more than two tasks simultaneously. Foreground and background tasks must also be seamlessly integrated to help minimise some of the inevitable performance limitations of current systems and to best exploit future, more capable systems. For example, the effective implementation of the hypermedia scenario described in Section 2 requires background tasks to be constantly operating to search for information and to update indexes.

\section{Standards Bodies}

The most important working groups producing standards for IS\&N are associated with ITU-T, ETSI and ISO/IEC. The flow of information between these groups is shown in Figure 4.

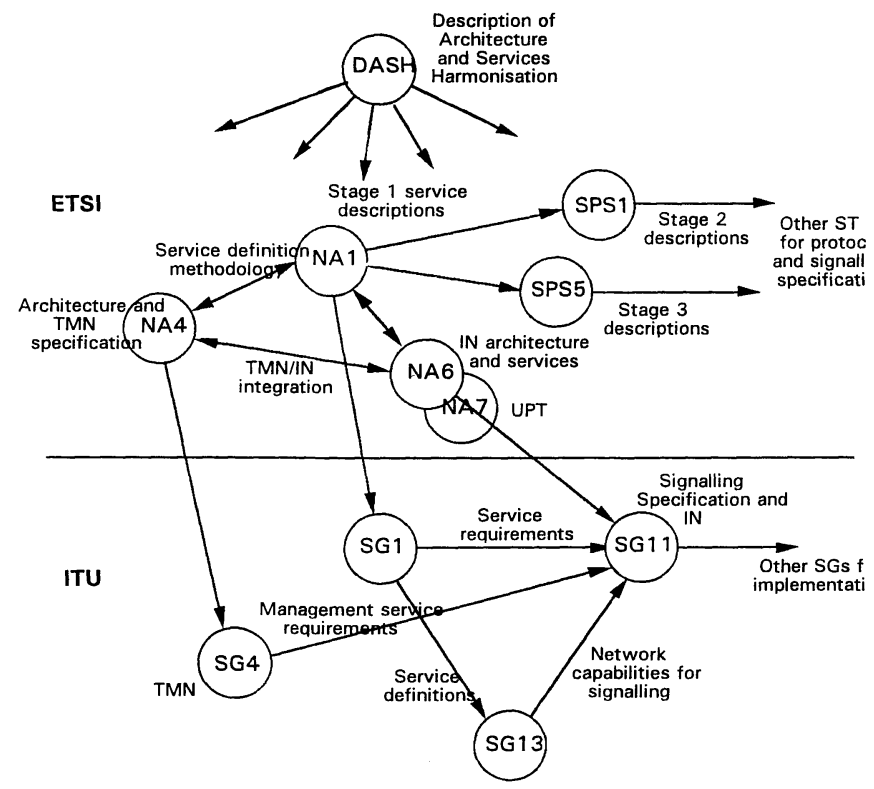

Figure 4 The relationship between the standards groups most important to IS\&N. 
There are also important standards produced by ISO/IEC and de-facto standards.

\subsection{European Telecommunications Standards Institute (ETSI)}

ETSI is European regional standards body and it the primary focus of work from RACE. ETSI produces Technical Standards (ETSs) and Technical Reports (ETRs). Neither of these are mandatory in their own right, but they can be referenced by the CEC in European Directives, which then become mandatory on all European equipment suppliers. Standards in ETSI are drafted by 11 Technical Committees (TCs), each divided into a number of Sub-Technical Committees (STCs). The most important TCs to IS\&N are:

NA Network Aspects are the core of the work in RACE towards IBC. It is necessary to contribute to NA to ensure that the current networks can evolve towards the seamless integrated broadband network(s) of the future. NA1 covers services, NA2 covers numbering and addressing, NA4 covers architectures and TMN, NA5 covers broadband, NA6 covers IN and NA7 covers UPT. All STCs are important to IS\&N.

SPS Signalling, Protocols \& Switching. Service and network capability requirements should be provided by NA to SPS as shown in Fig 4, so that the signalling and protocol specifications can evolve to meet the requirements of future services. However, signalling capabilities are often defined in advance of the services for which they are provided, and this will increasingly be the case for $\mathrm{IN}$ services where, to enable maximum flexibility of service provision, a full set of services are not defined before the signalling capabilities are implemented. All STCs within SPS are important, especially SPS3 which covers digital switching.

SMG Special Mobile Group. Mobile access is becoming increasingly important to users and must be integrated seamlessly into IBC. The most important STC is SMG5 which is currently specifying UMTS. SMG1 and SMG3 are also important.

\subsection{International Telecommunications Union (ITU)}

The ITU produces recommendations that are applicable worldwide and so must be addressed to enable global services to be provided. Work in the telecommunications standards sector of ITU is carried out in 15 Study Groups (SGs). The most important for ISN are:

SG1 Service Definition. A wide range of services are being defined including multimedia and multipoint conferencing services.

SG4 Network Maintenance. This includes all recommendations on the TMN.

SG11 Switching and Signalling. This includes all recommendations on IN.

SG13General Network Aspects. This includes the specification of B-ISDN and the specification of the network capabilities required to support multimedia services.

\subsection{International Organisation for Standardisation (ISO)}

ISO standards cover all fields except for electrical and electronic engineering which is covered by IEC, and telecommunications which is covered by ITU. The technical work of ISO is done in technical committees (TCs) and their subcommittees (SCs) and working groups (WGs). 
The work relevant to ISN is covered in joint groups with IEC. The most important of these are:

- ISO TP

- ISO CMIP/CMISE

\subsection{Other standards}

De facto standards such as X/Open, OMG and OSF, and emerging IT technologies such as DCE, OLTP, CORBA and Motif are a major influence in the TMN area. The Internet community has also been very successful in establishing de-facto standards for such things as routers and messaging systems. These were available before and are operating in competition to internationally recognised standards for systems with similar functionality.

\section{Conclusions}

This paper has listed the high priority areas in which work is needed. It is not suggested that all projects can or need to contribute to all the above areas. However, it is important that some means be found through the current management activities to better coordinate this joint effort. In particular, while efforts in TINA, EURESCOM and the CEC funded RACE/ACTS Programmes are essentially independent activities, it is important that there be a means to coordinate the effort in the interest not only of harmonised solutions, but also of more cost effective R\&D effort for the companies involved (both industry and operator).

\section{Glossary}

CCITTInternational Telegraph and Telephone Consultative Committee. The part of the ITU responsible for (non-mandatory) recommendations on public telecommunications services. CCITT publishes telecommunications recommendations in the form of books; the most recent is the Blue Book (1988).

ETSI European Telecommunications Standards Institute. A non-profit making organisation setting telecommunications standards in Europe. ETSI has 12 technical committees (TCs) dealing with telecommunications, IT (in cooperation with CEN) and broadcasting (in cooperation with the EBU).

ISO The International Organisation for Standardisation. A federation of national standards bodies. ISO sets worldwide standards in any field not covered by a specialist standards body.

ITU The International Telecommunications Union. An agency of the United Nations based in Geneva. It is responsible for telecommunications standards worldwide and has 5 parts including CCITT and CCIR. On 1 March 1993 CCITT and CCIR were merged into a single part of ITU responsible for telecommunications standards.

PSCS Personal Services Communications Space.

UMTSUniversal Mobile Telecommunications System.

UPT Universal Personal Telecommunications. 


\section{$8 \quad$ References}

[1] ETSI TCR-TR/NA-003001 "Recommendations towards the Harmonisation of Architecture and Service Description Methodologies".

[2] Standardisation in Information Technology and Telecommunications, Commission of the European Communities DG XIII: 200 rue de la Loi, B-1049 Brussels, Belgium.

[3] MOBILISE PSCS Concept: Definition and CFS - Draft Version. Deliverable 4, RACE Project R2003, June 1993.

[4] ETSI DTR/NA-10100 "UPT Phase 1 - Service Description".

[5] Report of workshop on UPT Management, Bonn, May 1994.

[6] Report of joint STG meeting of IS\&N STGs, STG JOI(94)1/R, Brussels, 18 May 1994.

[7] CCITT Recommendation I.130 "Method for the Characterisation of Telecommunication Services supported by an ISDN and Network Capabilities of an ISDN".

ISO standards can be obtained from the ISO Central Secretariat, 1, rue de Varembé, Case postale 56, CH-1211 Geneva 20, Switzerland.

CCITT Recommendations can be obtained from ITU Headquarters, Place des Nations, CH-1211, Geneva 20, Switzerland.

ETSI Technical Reports and ETSI Technical Standards can be obtained from the ETSI Secretariat, 06921 Sophia Antipolis Cedex, France. 